\title{
Surgery-as-soon-as-Feasible: A Challenge to One Major Premise of the Surgery-First Approach
}

\author{
Michael G Woods* \\ Oral and Maxillofacial Surgery unit, Royal Melbourne Hospital, Australia
}

*Corresponding author: Michael G Woods, Professor and consultant orthodontist, Oral and Maxillofacial Surgery unit, Royal Melbourne Hospital, Australia, Tel: +61407810210; E-mail: mgwoodsorthodontics@gmail.com

Received: 17 Mar, 2021 | Accepted: 24 Mar, 2021 | Published: 30 Mar, 2021

Citation: Woods MG (2021) Surgery-as-soon-as-Feasible: A Challenge to One Major Premise of the Surgery-First Approach. Int J Dent Oral Health 7(3): dx.doi.org/10.16966/2378-7090.359

Copyright: (02021 Woods MG. This is an open-access article distributed under the terms of the Creative Commons Attribution License, which permits unrestricted use, distribution, and reproduction in any medium, provided the original author and source are credited.

\begin{abstract}
Aim: The aim of this paper is to simply document individual pre and post-surgical durations in a large sample of orthognathic patients treated with an overall philosophy of Surgery-as-soon-as-feasible and to then compare the presurgical durations, especially, with claims of "exceptionally-long traditional presurgical treatment durations, of two years or more" made by some proponents of the so-called Surgery-first approach.

Method: The actual dates of placement of fixed appliances, definitive anteroposterior and vertical surgery and removal of fixed appliances were noted from the legally-stored records of 203 individual orthognathic surgery patients, all having undergone Surgery-as-soon-as-feasible. Individual presurgical and post-surgical durations were calculated. Mean changes in the total sample and various anteroposterior and vertical subgroups were then calculated and compared.

Results: The mean durations for the total sample and the subgroups were similar. In fact, there were no significant differences in those means. There were, however, considerable ranges of variation around all those means. When the patients in the total sample were viewed individually, it was obvious that many patients went to surgery very early in the braces period. In fact, of the 203 patients, 36 went to surgery within 6 months and 130 went within 12 months.

Conclusions: Presurgical treatment durations in these Surgery-as-soon-as-feasible patients were not generally "exceptionally-long, of two years or more." In fact, in many cases, patients underwent jaw surgery very early in their braces period. It seems that, depending upon the presenting dentofacial morphology, some presurgical durations will indeed be greater than one year - but that's not the rule. It will depend on the need for expansion, extractions and three-dimensional control in particular anteroposterior and vertical patterns. Carrying-out early surgery in wellthought-out situations (whether or not it is labelled as "Surgery-first") is entirely appropriate. That is not inconsistent though with the overall long-term approach of treating all individual orthognathic patients with Surgery-as-soon-as-feasible. It depends on the needs of the particular set of dentofacial problems.
\end{abstract}

\section{Introduction}

The so-called Surgery-first approach (usually involving sophisticated three-dimensional digital planning and the use of skeletal screws and plates as orthodontic anchors for necessary tooth movements) has been promoted to reduce "exceptionallylong presurgical orthodontic treatment durations of two years or more" [1]. It is also promoted on the basis that the so-called Rapid Acceleratory Phenomenon (the RAP) effect) following bone trauma or surgery allows the teeth to move through bone more quickly-in the end, leading to much-reduced total active treatment durations $[1,2]$. It has been pointed out however that, despite the use of skeletal orthodontic anchorage, presenting dentoalveolar compensations may not always be completely reduced [3]. It has also been pointed out that the Surgery-first approach may not be appropriate anyway for all patients being considered for three-dimensional orthognathic surgery [4]. In the hands of Surgery-first-experienced orthodontists and surgeons, the published results of treatment appear to be very goodfor the surgical correction of a number of (but not all) dentofacial conditions [5].

Since the 1990s, this author and his colleagues have written a number of papers promoting a Surgery-as-soon-as-feasible approach, with surgical/orthodontic treatment and timing for all patients based on individual morphological characteristics and the need in all cases to deal appropriately with three-dimensional dentoalveolar compensations [6-13]. Some presurgical treatment times have been very short (1 to 6 months), most are reasonable (7 to 11 months) and a few have been longer (12 to 18 months). Most post-surgical treatment times have been close to 6 months. The key though has 
been to move all patients through Surgery-as-soon-as-feasible as efficiently as possible (also acknowledging an expected increase in bone turnover and rapidity of tooth movement in the immediate postsurgical period) [7-9].

Keeping in mind, the published premise that traditional orthognathic surgery routinely involves "long presurgical treatment durations of two years or more", the aim of this paper is to simply present the actual documented pre and post-treatment durations for all patients in a group of varied subjects, treated with Surgery-as-soonas-feasible in Melbourne, Australia.

\section{Method}

\section{The sample}

Documented pre and post-surgical treatment durations were calculated from the legally-stored records of 203 late-teenage and young-adult patients, with various dentofacial conditions, all treated with a Surgery-as-soon-as-feasible approach. This simply means that these patients started orthodontic treatment and were taken to surgery as soon as deemed ready for the safe and predictable management of their particular dentofacial problems. Some patients went very early; others went later. Of the total sample of 203 patients, 142 were female and 61 were male. These patients were those included in a parallel study of skeletal and dental changes occurring in the longer term after treatment $[14,15]$. (The total sample for the previous study had been divided into four groups-on the basis of vertical type (average $\mathrm{FMP}=25$ degrees) and the anteroposterior direction in which the surgery had been carried out). All patients or their families had signed informed-consent forms prior to the commencement of their surgical and orthodontic treatment. Those forms included permission to use their anonymous treatment records for teaching, publication and/ or professional presentation. The duration numbers for this current paper are also presented as the total sample divided into those same four groups. This "low-risk" project was the subject of approval by the Latrobe University Human Research Ethics committee (\#HEC 20434).

\section{The treatment}

All patients had been treated by the same orthodontist and various senior consultant surgeons. Routine contemporary fixed appliances, with or without extractions, elastics and/or maxillary expansion, were used to treat the patients in this group [8]. All these stages are included in the documented total presurgical duration. Differences in underlying vertical pattern were really important in all decisionmaking [16-18]. The aims of the presurgical orthodontic preparation have always been to address the dentoalveolar compensations in all three dimensions, with some plan for management in the transverse dimension, also placing the teeth in inter-arch relationships which reflect similar anteroposterior needs at the canines and the incisors.

With Surgery-as-soon-as-feasible, the bias is to complete as much dentoalveolar movement as possible before surgery in long-face, openbite types. In all other cases, however, only tooth movements necessary to facilitate the ideal surgical jaw movements are carried out [9]. The thought process has always been to "unlock" the hostile pre-treatment musculoskeletal occlusion as soon as possible and to use the individual mandibular muscle pattern and the expected increased bone turnover (RAP) to allow the occlusion to be efficiently and carefully detailed [7].

\section{Gathering of the data}

The dates of initial placement of orthodontic fixed appliances, actual definitive anteroposterior and vertical orthognathic surgery and the removal of fixed appliances were all taken directly from the legally- stored treatment records of all patients in the sample. The presurgical and post-surgical treatment durations were then simply calculated in months, for all individual patients. The total sample had been divided into Class II and Class III surgical groups on the basis of the anteroposterior direction in which the individual surgical movements had been carried out $[14,15]$. The total sample was further divided into four subgroups on the basis of the pretreatment Frankfort-mandibular plane angle (FMA) (Table 1).

Finally, the four anteroposterior and vertical subgroups were each further divided according to percentages within the range of presurgical durations for that group (Table 2). Those with the presurgical durations in the lowest $20 \%$ of the range in each group; those with presurgical durations in the next $30 \%$ of the range (20-50); those with presurgical durations in the next $30 \%$ of the range (50 to 80 ); those with presurgical durations in the highest $20 \%$ of the range. For the purposes of this demonstration, it was considered that there was no need for the use of sophisticated statistics.

Means and standard deviations were then calculated (Table 1). Simple ANOVA was used to compare the mean durations of the various groups $(\mathrm{p}<0.05)$.

\section{Results}

All 203 individual pre and post-surgical treatment durations are illustrated in figure 1. From the figure, it can be seen that 36 patients had documented presurgical durations of less than 6 months and 130 had presurgical durations of less than 12 months.

Mean durations for the four anteroposterior and vertical subgroups are presented in table 1 . From the table, it can be seen that there were no significant differences in mean presurgical durations for the total sample or its four subgroups. The means all reflected presurgical durations of approximately 10 months. There were however large standard deviations (within one standard deviation, from approximately 5 to 15 months in all subgroups). The differences amongst the means for the total sample and the four subgroups were not found to be statistically significant $(\mathrm{p}<0.05)$.

Mean durations for the further subgroups within in the four main anteroposterior and vertical groups are presented in table 2. From the table, it can be seen that the lowest $20 \%$ of the subgroups all showed mean presurgical durations of 1 to 5 months. The next $30 \%$ had presurgical durations of 7 to 10 months, the next $30 \%-11$ to 15 months and those in the highest $20 \%$ group- 17 to 19 months.

Pre and post-treatment records of one individual patient are shown in figure 2 . This patient went straight to surgery after braces placement-

Table 1: Mean durations and standard deviations (months).

\begin{tabular}{|l|c|c|c|c|}
\hline \multicolumn{1}{|c|}{ Sample } & N & Pre-surg & Post-surg & Total \\
\hline Total & 203 & $10.5(5.0) N S$ & $5.9(1.4) \mathrm{NS}$ & 16.5 \\
\hline Class II longer & 79 & $10.8(4.9) \mathrm{NS}$ & $5.8(1.4) \mathrm{NS}$ & 16.4 \\
\hline Class II shorter & 55 & $10.9(5.0) \mathrm{NS}$ & $6.1(1.5) \mathrm{NS}$ & 17 \\
\hline Class III longer & 37 & $10.2(4.9) \mathrm{NS}$ & $5.8(1.2) \mathrm{NS}$ & 15.9 \\
\hline Class III shorter & 32 & $10.2(4.9) \mathrm{NS}$ & $5.8(1.2) \mathrm{NS}$ & 16.3 \\
\hline
\end{tabular}

The means might all be similar, but the standard deviations reflect wide individual variation, with some quite short presurgical duration and other considerably longer presurgical durations.

NS: Not significant $p<0.05$. The means were not significantly different to each-other. 
Table 2: Mean durations and Standard deviations (months).

\begin{tabular}{|c|c|c|c|c|}
\hline Duration Groups & $\mathbf{N}$ & Pre-surg & Post-surg & Total \\
\hline \multicolumn{5}{|c|}{ Total } \\
\hline$<20 \%$ & 36 & $3.2(1.7)$ & $6.3(1.6)$ & 9.3 \\
\hline $20-50 \%$ & 75 & $8.7(1.5)$ & $5.9(0.8)$ & 14.6 \\
\hline $50-80 \%$ & 65 & $13.6(1.5)$ & $5.8(1.1)$ & 19.4 \\
\hline$>80 \%$ & 27 & $18.0(0.9)$ & $5.9(0.9)$ & 23.9 \\
\hline \multicolumn{5}{|c|}{ Cl II Longer } \\
\hline$<20 \%$ & 14 & $3.1(1.8)$ & $5.6(1.8)$ & 8.8 \\
\hline $20-50 \%$ & 27 & $8.8(1.4)$ & $6.0(1.5)$ & 14.8 \\
\hline $50-80 \%$ & 27 & $13.4(1.5)$ & $5.8(1.0)$ & 19.8 \\
\hline$>80 \%$ & 11 & $17.8(1.0)$ & $6.0(1.8)$ & 23.8 \\
\hline \multicolumn{5}{|c|}{ Cl II Shorter } \\
\hline$<20 \%$ & 9 & $3.1(2.0)$ & $7.2(1.5)$ & 10.3 \\
\hline $20-50 \%$ & 21 & $8.9(1.8)$ & $6.1(1.4)$ & 15.1 \\
\hline $50-80 \%$ & 17 & $14.1(1.1)$ & $5.5(1.6)$ & 19.6 \\
\hline$>80 \%$ & 8 & $18.9(1.2)$ & $6.0(0.8)$ & 24.9 \\
\hline \multicolumn{5}{|c|}{ Cl III Longer } \\
\hline$<20 \%$ & 7 & $2.7(1.8)$ & $6.1(1.3)$ & 10.3 \\
\hline $20-50 \%$ & 14 & $8.5(1.5)$ & $5.4(1.2)$ & 13.9 \\
\hline $50-80 \%$ & 11 & $13.3(1.6)$ & $6.1(1.3)$ & 18.8 \\
\hline$>80 \%$ & 5 & $17.8(0.8)$ & $5.6(1.1)$ & 23.4 \\
\hline \multicolumn{5}{|c|}{ Cl III Shorter } \\
\hline$<20 \%$ & 6 & $2.8(1.3)$ & $6.8(1.2)$ & 9.7 \\
\hline $20-50 \%$ & 12 & $8.4(1.2)$ & $6.1(1.2)$ & 14.5 \\
\hline $50-80 \%$ & 10 & $13.3(1.6)$ & $6.1(1.5)$ & 19.4 \\
\hline$>80 \%$ & 4 & $17.8(0.9)$ & $6.0(0.8)$ & 23.8 \\
\hline
\end{tabular}

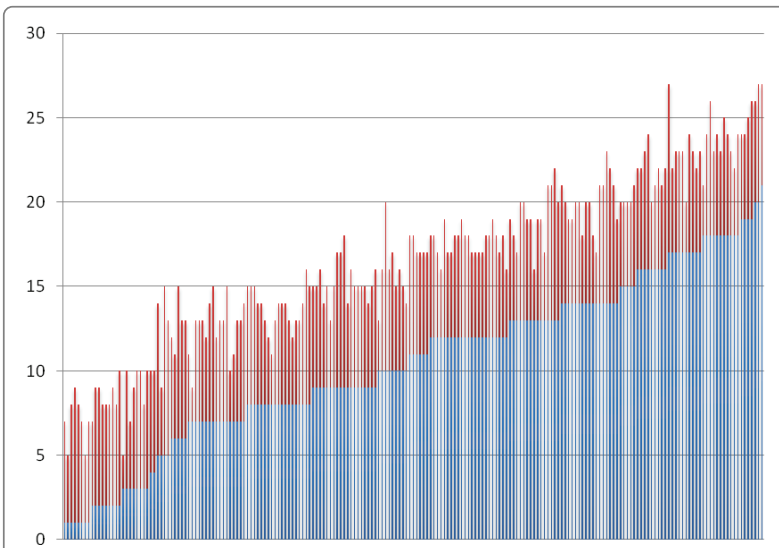

Figure 1: Pre and post-surgical treatment durations (months) for all 203 individual Surgery-as-soon-as-feasible patients.

Blue columns-Presurgical; Pink columns-Post-surgical. (For 36 patients $<6$ months presurgical; For 130 patients, <12 months Presurgical). not because of an "early surgery" philosophy, but simply because that was all that was necessary in that case.

\section{Discussion}

These actual durations, taken directly from legally-stored patient records, reflect the fact that, as with most other morphological and/ or treatment questions in orthodontics and/or orthognathic surgery, individual variation and individual needs are the rules rather than the exceptions. This means that, only once detailed individual dentofacial aims have been set, can the appropriate treatment decisions be made. This applies to the choice of devices, timing, the need for expansion, extractions and orthognathic surgery, and would be the case regardless of whether manual two-dimensional or sophisticated digital threedimensional planning were to be undertaken.

When reviewing this discrete sample of Surgery-as-soon-as-feasible patients, whose surgery was carried out consistently over a number of years, the aims have obviously been to provide ideal facial balance and well-functioning occlusion with pleasing lateral facial and anterior smile balance. Patients have received treatment aimed at properly dealing with dental compensations in all three dimensions. Rapid palatal expansion or preliminary surgically-assisted RPE have been undertaken, as necessary [8]. Premolars were extracted in some cases to allow either or both upper or lower anterior teeth to be aligned and moved into pre-planned, presurgical positions. Rigid internal screw and plate fixation was used in all these cases. Within one to two weeks after surgery, all patients were back in the hands of the orthodontist.

The mean presurgical durations found in this sample might all be similar, but the standard deviations reflect very wide individual variation, with some quite short presurgical durations and other considerably longer presurgical durations. The aim of Surgery-assoon-as-feasible is to move all patients through surgery as efficiently as possible-the presurgical durations are likely to be different-mainly determined by their presenting dentofacial morphology, the need to eventually deal with all three-dimensional dental compensations and to respect the individual underlying muscular patterns. The socalled Rapid Acceleratory Phenomenon (the RAP effect) is very much considered when deciding when the appropriate amount of presurgical orthodontic preparation has been achieved [7,9].

The use of skeletal orthodontic anchorage is acknowledged to be effective in controlling movements of individual teeth, or groups of teeth-or in fact entire arches in certain surgical and non-surgical situations $[1,5]$. Three-dimensional imaging, planning and treatment technology will continue to be developed to the advantage of all those involved in all types of orthognathic treatment [2,18]. By all accounts, in skilled hands, Surgery-first has been shown to be an effective way in which various dentofacial problems can be managed [4]. It has also been acknowledged by its proponents, however that, at this stage, it is not necessarily appropriate for management of all surgical cases [5]. Like Surgery-as-soon-as-feasible, Surgery-first may involve extractions and/or expansion. There may also be the need for extra segmental surgery and an extra operation for placement of the orthodontic skeletal anchorage screws and plates. This author and his surgical colleagues have also been undertaking preliminary surgicallyassisted expansion operations, as necessary, for 30 years [8].

The traditional surgical/orthodontic textbooks [19] have often presented the aim of presurgical orthodontics as trying to achieve ideal dentoalveolar control before surgery (perhaps that has led to the perception of "exceptionally-long"). In this way, the best occlusion would be achieved at surgery. For those treating many surgical cases, however, it quickly became obvious that the achievement 

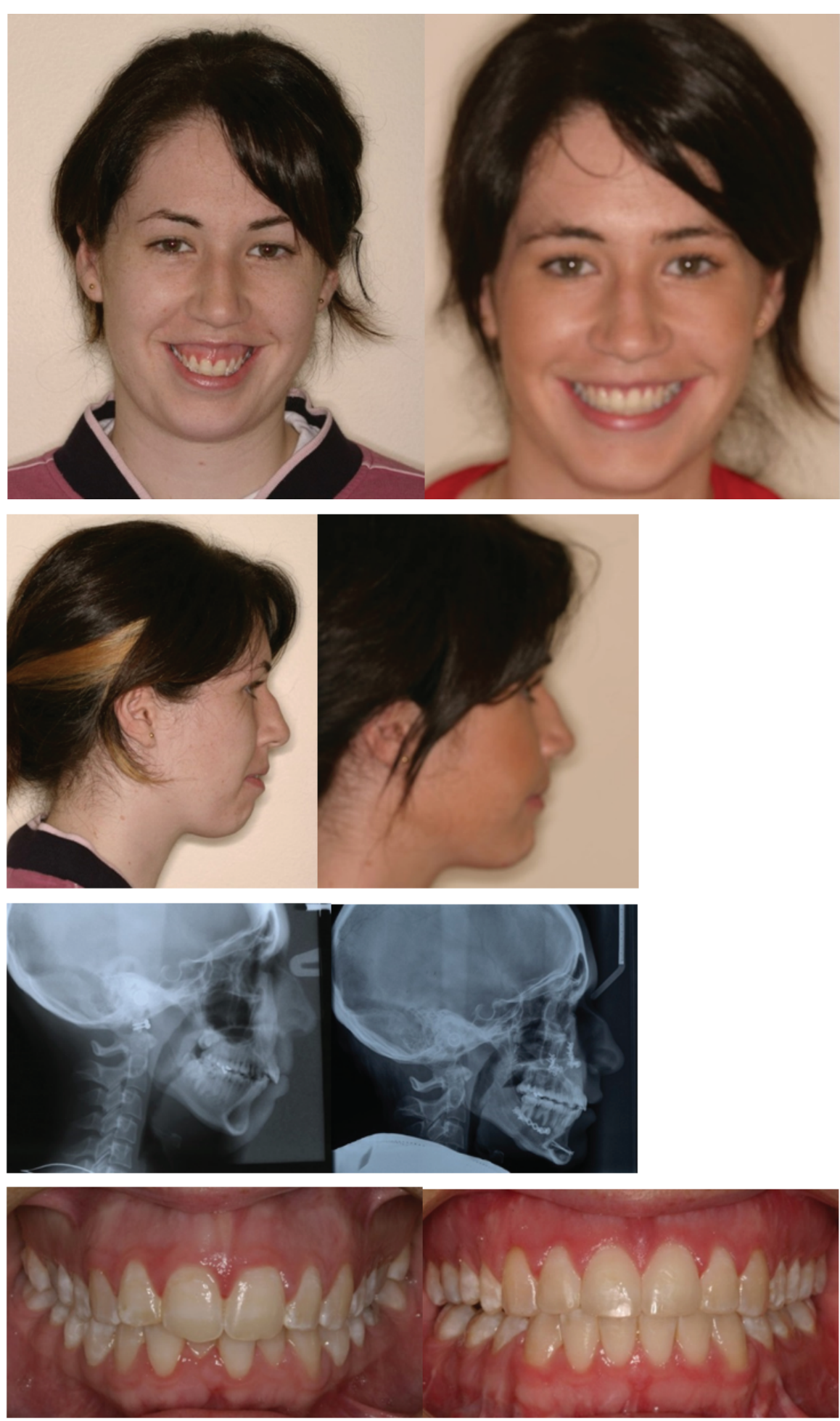

(a)

(b)

Figure 2: 22 year old female (frontal and lateral facial photographs, lateral cephalograms, and anterior dentition).

(a) Pretreatment records-fixed appliances placed (presurgical duration-1 month, straight to surgery). That was appropriate in this case-it doesn't have to labelled with "Surgery-first" or anything else. (Maxillary impaction, autorotation, mandibular advancement, anterior sliding genioplasty, courtesy: Mr S Gibbons, oral and maxillofacial surgeon).

(b) Post-treatment records (post-surgical duration-5 months). 
of dentoalveolar perfection was not always possible in the hostile presurgical muscular and occlusal environment. The most important difference seemed to be the effect of different underlying vertical muscular patterns [17]. In long-face, open-bite types, as much presurgical detailing as possible has been done-so that after surgery, as little as possible dental extrusion (and posterior mandibular rotation) would be allowed to occur. In all other cases, however, only the absolutely necessary movements have been carried out before surgery [9]. No public hospital waiting lists were involved in this sample, with all patients receiving their private practice jaw operations as soon as they were deemed to be ready. There was generally no concern in leaving immediate post-surgical premolar and first molar open bites, with only anterior and posterior second molar occlusal stops. These patients have always been treated with the expectation that there would be increased bone turnover (and more rapid tooth movement) during the immediate post-traumatic healing period. It has also allowed the occlusions to be carefully detailed in three dimensions in the "newlyunlocked" post-surgical environment.

\section{Conclusions}

From the above assessment of treatment durations in this sample of 203 patients, the following conclusions can be drawn:

- $\quad$ Presurgical treatment durations in these Surgery-as-soonas-feasible patients were not generally "exceptionally-long, of two years or more." In fact, in many cases, patients underwent jaw surgery very early in their braces period.

- It seems that, depending upon the presenting dentofacial morphology, some presurgical durations will indeed be greater than one year-but that's not the rule. It will depend on the need for expansion, extractions and three-dimensional control in particular anteroposterior and vertical patterns [9].

Like many relatively-new techniques, devices, and regimes, Surgery-first is likely to increase in popularity and will then probably settle-in for the long term as one valuable part of the orthodontic and surgical armamentarium. Carrying-out early surgery (whether or not it is labelled as "Surgery-first") in well-thought-out situations is entirely appropriate. That is not inconsistent though with the overall long-term approach of treating all individual orthognathic patients with Surgery-as-soon-as-feasible.

\section{Acknowledgements}

The author would like to thank all the Royal Melbourne Hospital Oral and Maxillofacial Surgery consultants for their management of the patients included in this sample.

\section{Conflict of Interest}

No conflict of interest.

\section{Funding}

This work was supported within a general research grant from the Australian Society of Orthodontists' Foundation for Research and Education.

\section{Ethical Approval}

Latrobe University Human Research Ethics approval \#HEC 20434.

\section{References}

1. Villegas C, Uribe F, Sugawara J, Nanda R (2010) Expedited correction of significant dentofacial asymmetry using a "surgery-first" approach. J Clin Orthod 44: 97-103.
2. Kim JK, Park JH, Jung HD, Jung YS (2020) Factors affecting total treatment time in patients treated with orthognathic surgery using the Surgery-first approach: multivariable analysis using 3D CT and scanned dental casts. J Clin Med 9: 641.

3. Yu HB, Mao LX, Wang XD, Fang B, Shen SG (2015) The surgery-first approach in orthognathic surgery: a retrospective study of 50 cases. Int J Oral Maxillofac Surg 44: 1463-1467.

4. Uribe F, Agarwal S, Shafer D, Nanda R (2015) Increasing orthodontic and orthognathic surgery treatment efficiency with a modified surgery-first approach. Am J Orthod Dentofac Orthop 148: 838-848.

5. Gandedkar NH, Dávila MMC, Chng CK, Liou EJW, Darendeliler A (2019) Surgery-first orthognathic approach: A "scoping review" for mapping outcomes and plausible recommendations to develop core outcome sets. APOS Trends Orthod 9: 77-88.

6. Kirkpatrick TB, Woods MG, Swift JQ, Markowitz NR (1987) Skeletal stability following mandibular advancement and rigid fixation. J Oral Maxillofac Surg 45: 572-576.

7. Woods MG, Swift JQ, Markowitz NR (1989) Clinical implications of advances in orthognathic surgery. J Clin Orthod 23: 420-429.

8. Woods MG, Wiesenfeld D, Probert TCS (1997) Surgically-assisted maxillary expansion. Aust Dent J 42: 38-42.

9. Woods MG, Wiesenfeld D (1998) A practical approach to presurgical orthodontic preparation. J Clin Orthod 32: 350-358.

10. Baker BW, Woods MG (2001) The role of the divine proportion in the esthetic improvement of patients undergoing combined orthodontic/orthognathic surgical treatment. Int J Adult Orthod Orthognath Surg 16: 108-120.

11. Woods MG (2002) Class III treatment opportunities. Ann Roy Australas Coll Dent Surg 16: 71-73.

12. Shell TL, Woods MG (2003) Perception of facial aesthetics: A comparison of similar Class II cases treated with attempted growthmodification or later orthognathic surgery. Angle Orthod 73: 365373.

13. Georgalis K, Woods MG (2015) A study of Class III treatment: orthodontic camouflage vs orthognathic surgery. Aust Orthod J 31: 138-148.

14. Woods MG (2016) 10 year+ follow-up vertical changes after contemporary Class II and III orthognathic surgery. Eur Acad Cranio Maxillo Fac Surg $23^{\text {rd }}$ Congress, London, Abstr, EACMFS-904.

15. Woods MG (2020) Surgery-as-soon-as-feasible: a challenge to a major premise of the Surgery-first approach. World Fed Orthods, $9^{\text {th }}$ International Orthodontic Congress, Yokohama, Abstr WFO-20671.

16. Pepicelli AJ, Woods MG, Briggs C (2005) The mandibular muscles and their importance in contemporary orthodontics: A review. Am J Orthod Dentofac Orthop 128: 774-780.

17. Woods MG (2017) The mandibular muscles in contemporary orthodontic practice: a review. Aust Dent J 62: 78-85.

18. Mastroianni D, Woods MG (2019) 3D CT assessment of mandibular widths in young subjects with different underlying vertical patterns. J World Fed Orthod 8: 78-86.

19. Proffit WR, Sarver DM (2018) Combined Surgical and orthodontic treatment. In: Fields HW, Larson BE, Sarver DM (eds) Contemporary Orthodontics. $6^{\text {th }}$ Edition, Chapter 20, Elsevier, Philadelphia, PA 657692. 